Classification

Physics Abstracts

05.40

\title{
Field theory approach to the Eden model and diffusion-limited aggregation
}

\author{
L. Peliti \\ Dipartimento di Fisica, Università « La Sapienza », Piazzale Aldo Moro 2, I-00185 Roma, Italy \\ and GNSM-CNR, Unità di Roma \\ and Zhang Yi-Cheng (*) \\ Physics Department, Brookhaven National Laboratory, Upton, N.Y. 11973, U.S.A.
}

(Reçu le 25 juin 1985, accepté sous forme définitive le 21 octobre 1985)

\begin{abstract}
Résumé. - On montre que le formalisme d'espace de Fock pour des objets classiques, dû à Doi et à Grassberger-Scheunert, permet d'écrire systématiquement la théorie des champs pour des processus d'agrégation tels que le modèle d'Eden et l'agrégation limitée par diffusion. On discute brièvement de la relation de ces théories avec la théorie de champs pour les Reggeons. On suggère une origine possible des difficultés rencontrées dans l'application des méthodes du groupe de renormalisation à ces problèmes.

Abstract. - We show that the Fock space formalism for classical objects, due to Doi and GrassbergerScheunert, allows us to write down systematically field theories for aggregation processes such as the Eden model and diffusion-limited aggregation. The relation of such theories with Reggeon field theories is briefly discussed. The possible origin of difficulties in applying renormalization group methods to these problems is hinted.
\end{abstract}

\section{Introduction.}

Kinetic aggregation models are presently the object of intensive investigation. The most popular models of this kind are the Eden model [1] and the Witten-Sander model of diffusion-limited aggregation (DLA) [2]. A large part of this investigation is carried out by computer simulation [3], although different theoretical approaches are not lacking. A few exact results for the Eden and DLA models in the limit of many dimensions have indeed been obtained by Parisi and Zhang [4] and by Vannimenus et al. [5]. More recently, the geometrical characteristics of the aggregates have been put in relation with the growth mechanism of DLA by Pietronero [6], Turkevitch and Sher [7], and Halsey et al. [8].

(*) Supported by U.S. Department of Energy, under contract No. DE-ACO2-76CH00016. 
One of the main purposes of current research is to identify the mechanism at the origin of the remarkable self-similarity of the aggregates and a way to calculate their fractal dimension. The success obtained in carrying out a similar program for critical fluctuations has led to the expectation that this goal could be reached by means of renormalization group ( $R G)$ methods, possibly in its field theoretical formulation [9], whose computational power is well known. Indeed, a few theoretical approaches to birth-death processes on a lattice, closely resembling aggregation models, have appeared in the literature. The most popular model of this kind is Schlögl's first model [10] - a model of a self-controlled chemical reaction, which is also related to directed percolation. Such a model has been treated by field theoretical techniques, closely related to Reggeon Field Theory (RFT : see e.g. Amati et al. [11]) by several authors [12-14]. More recently, field theoretical approaches to a few birth-death processes with diffusion have also been considered. Cardy [15] has introduced immunization effects in Schlögl's model and has treated the corresponding non-Markovian process by a field theory obtained along the lines of that describing the " true " self-avoiding walk [16]. This analysis has been reexamined by Cardy and Grassberger [17] and by Janssen [18], who came to the conclusion that the static properties of this model are in the same universality class as the statistics of percolation clusters. Further information, concerning in particular the spreading dimension of such clusters, could therefore be obtained.

On the other hand, Parisi and Zhang [19] have introduced, on the basis of heuristic considerations, field theories describing the Eden and DLA model. Their conclusion is that the Eden model corresponds to a RFT with local interactions, deep in the ordered region, whereas DLA corresponds to a generalized RFT with nonlocal interactions. Shapir and Zhang [20] have considered a systematic Hamiltonian approach to the Eden model, deriving a field theory which is local in space, but nonlocal (non-Markovian) in time. When this work was being completed, we received a preprint by Shapir [21] containing similar results based on a slightly different approach.

What is striking in these works is the variety of techniques used to reach essentially similar results. Methods range from the introduction of suitable operator algebras, to the use of Langevin equations, to the consideration of a hierarchy of the BBGKY type. It is as if new techniques had to be forged from scratch each time.

The aim of this paper is to show that a single well cut tool is sufficient to derive field theoretical descriptions of Eden and DLA models in a systematic way. The tool is the Fock space formalism for classical objects, first introduced by Doi [22] and then reformulated by Grassberger and Scheunert [23]. (Reference [24] contains a pedagogical introduction). It leads to a nontrivial generalization of the Martin-Siggia-Rose (MSR) formalism for classical evolution equations [25]. While its application solves the kinematical problem of finding the correct field theory, one still faces the dynamical problem of its RG analysis. One notices in fact that RG techniques have so far met with success only in the case of Schlögl's models, without or with immunization, which correspond to directed and ordinary percolation respectively. One can identify in these cases an upper critical dimension $d_{\mathrm{c}}$ above which the process essentially behaves like ordinary diffusion. The scaling behaviour of the process can then be obtained in an expansion around $d_{c}$, making use of a fixed point of RG transformations, which can be reached within perturbation theory. In the case of our models one notices instead the instability of the trivial fixed point corresponding to diffusion at any dimension. As a consequence, perturbation theory is not able to provide us with a stage where to implement RG transformations. One may hope that this is just a technical difficulty, and that a different formulation (building perhaps on exact solutions of nonlinear field theories) may yield a " good " fixed point around which to expand. There are however hints that what is needed goes much beyond this, and that no less than a reformulation of the whole RG philosophy is necessary.

The Fock space formalism for classical objects is sketched in section 2 . The field theories of the Eden model are derived in section 3, and their relations with RFT are briefly discussed. Section 4 contains a derivation of the field theory of DLA. Section 5 contains some considerations about the difficulties encountered in implementing the $\mathrm{RG}$ program for these theories. 


\section{Fock space formalism for classical objects.}

This formalism [22-24] makes it possible to write down systematically field theoretical descriptions of birth-death processes on a lattice. Let the microscopic state $n$ of the system be identified by the set $\mathbf{n}=\left\{n_{\mathbf{r}, \alpha}\right\}$ of occupation numbers of particles of species $\alpha$ at the site $\mathbf{r}$ of a $d$-dimensional lattice. The macroscopic state $|\phi\rangle$ is then identified by the probability $\phi(n)$ of finding the system in the microscopic state $|n\rangle$. All states are considered as elements of a Hilbert space, and in particular one has :

$$
|\phi\rangle=\sum_{n} \phi(n)|n\rangle .
$$

The evolution of the state $|\phi\rangle$ is given by a master equation of the form :

$$
\frac{\mathrm{d}|\phi\rangle}{\mathrm{d} t}=L|\phi\rangle
$$

where the Liouvillian operator $L$ is expressed in terms, of the annihilation $a_{\mathbf{r}, \alpha}$ and creation $\pi_{\mathbf{r}, \alpha}$ operators defined by :

$$
\begin{gathered}
a_{\mathbf{r}, \alpha}\left|\ldots, n_{\mathbf{r}, \alpha}, \ldots\right\rangle=n_{\mathbf{r}, \alpha}\left|\ldots, n_{\mathbf{r}, \alpha}-1, \ldots\right\rangle, \\
\pi_{\mathbf{r}, \alpha}\left|\ldots, n_{\mathbf{r}, \alpha}, \ldots\right\rangle=\left|\ldots, n_{\mathbf{r}, \alpha}+1, \ldots\right\rangle .
\end{gathered}
$$

These operators satisfy the usual commutation relations :

$$
\left[a_{\mathrm{r}, \infty} \pi_{\mathbf{r}^{\prime}, \alpha^{\prime}}\right]=\delta_{\mathbf{r r}^{\prime}} \delta_{\alpha \alpha^{\prime}} .
$$

The Hilbert space structure is introduced in such a way that the Hermitian conjugate $a_{\mathbf{r}, \alpha}^{+}$of the annihilation operator is given by :

$$
a_{\mathrm{r}, \alpha}^{+}=\pi_{\mathrm{r}, \alpha}-1 .
$$

This corresponds to the « inclusive » scalar product of Grassberger and Scheunert [23]. One can then apply standard manipulations to solve equation (2) by means of path integrals (see e.g. Ref. [26]). One thus obtains a field theoretical model of the MSR kind, identified by the Lagrangian

$$
\mathfrak{L}=\sum_{\mathbf{r}, \alpha} \bar{\psi}_{\mathbf{r}, \alpha} \dot{\psi}_{\mathbf{r}, \alpha}-\mathfrak{L}[\bar{\psi}, \psi],
$$

where the classical fields $\bar{\psi}$ (imaginary), $\psi\left(\right.$ real) have taken the place of the operators $a^{+}, a$ respectively. In going from equation (2) to equation (7) one should take care to express the Liouvillian $L$ in terms of normal products, with all annihilation operators on the right of the creation ones. The Lagrangian (7) is the starting point of perturbation theory. One separates its « free » part, bilinear in $\bar{\psi}, \psi$, from the rest, which is then treated as a perturbation. It is usually possible to simplify drastically the model by going at once to the continuum limit.

\section{The Eden model.}

The Eden model [1] is usually defined as follows. At epoch $t_{0}=0 \mathrm{a}$ particle is placed at the origin of a (simple cubic) $d$-dimensional lattice; at epoch $t$, a new particle is added at one of the empty neighbours of the first particle. The process goes on, by adding at epoch $t_{n+1}$ a particle at one of the empty neighbours of the aggregate formed by the $n$ particles present at epoch $t_{n}$. We shall modify this definition by assuming instead that each member of the aggregate may add a particle 
at one of its neighbouring sites, if it is empty, with a certain rate $\mu$. The " time " of the original Eden model is thus proportional to the aggregate mass in this formulation. If one neglects the constraint, one obtains the " penetrable » Eden model, which is exactly solvable and which we shall discuss first.

The penetrable Eden model is therefore defined by the following reaction :

$$
\mathrm{X}_{\mathrm{r}} \stackrel{\mu}{\longrightarrow} \mathrm{X}_{\mathbf{r}}+\mathrm{X}_{\mathrm{r}+\mathrm{e}}
$$

where $\mathbf{r}$ is any lattice point and $\mathbf{r}+\mathbf{e}$ any of its nearest neighbours. The Liouvillian of this reaction is :

$$
L=\sum_{\mathbf{r}} \sum_{\mathbf{e}} \mu \pi_{\mathbf{r}}\left(\pi_{\mathbf{r}+\mathbf{e}}-1\right) a_{\mathbf{r}}
$$

One thus obtains the Lagrangian

$$
\mathcal{L}=\sum_{\mathbf{r}}\left\{\bar{\psi}_{\mathbf{r}} \dot{\psi}_{\mathbf{r}}-\mu \sum_{\mathbf{e}}\left(1+\bar{\psi}_{\mathbf{r}+\mathbf{e}}\right) \bar{\psi}_{\mathbf{r}+\mathbf{e}} \psi_{\mathbf{r}}\right\} .
$$

In view of the linearity of $\mathcal{L}$ with respect to $\psi$, the model can be solved exactly. One first integrates by parts the first term of equation (10) with respect to time and one then performs the functional integrations with respect to $\psi$, obtaining a deterministic evolution equation for $\bar{\psi}$ :

$$
\frac{\mathrm{d} \bar{\psi}_{\mathbf{r}}}{\mathrm{d} t}=-\mu \sum_{\mathbf{e}}\left(1+\bar{\psi}_{\mathbf{r}}\right) \bar{\psi}_{\mathbf{r}+\mathbf{e}} .
$$

All statistical properties of the model can be expressed in terms of the solution of this deterministic equation. The model can be then considered to be solved in principle.

The « real " Eden model can be obtained from this one by introducing a mechanism controlling the population growth. The easiest way is by introducing the reaction :

$$
X_{\mathbf{r}}+\mathbf{X}_{\mathbf{r}} \stackrel{v}{\longrightarrow} \mathbf{X}_{\mathbf{r}}
$$

We obtain therefore the Lagrangian :

$$
\mathcal{L}=\sum_{\mathbf{r}}\left\{\bar{\psi}_{\mathbf{r}} \dot{\psi}_{\mathbf{r}}+v\left(1+\bar{\psi}_{\mathbf{r}}\right) \bar{\psi}_{\mathbf{r}} \psi_{\mathbf{r}}^{2}-\mu \sum_{\mathbf{e}}\left(1+\bar{\psi}_{\mathbf{r}}\right) \bar{\psi}_{\mathbf{r}+\mathbf{e}} \psi_{\mathbf{r}}\right\}
$$

Let us order this Lagrangian with respect to the powers of the fields and their derivatives :

$$
\mathcal{L}=\sum_{\mathbf{r}}\left\{\bar{\psi}_{\mathbf{r}} \dot{\psi}_{\mathbf{r}}-\mu q \bar{\psi}_{\mathbf{r}} \psi_{\mathbf{r}}-\mu \Delta \bar{\psi}_{\mathbf{r}} \psi_{\mathbf{r}}+\nu \bar{\psi}_{\mathbf{r}} \psi_{\mathbf{r}}^{2}-\mu q \bar{\psi}_{\mathbf{r}}^{2} \psi_{\mathbf{r}}+\text { higher order terms }\right\} .
$$

We denote by $q$ the coordination number of the lattice and by $\Delta$ the discrete Laplacian. The first two lines reproduce the Lagrangian of RFT in the continuum limit, with a negative value of the " mass " (which is given here by $-\mu q$ ) ensuring the steady increase of the aggregate size. A similar result is obtained by considering instead the Liouvillian

$$
L=\mu \sum_{\mathbf{r}}\left\{\sum_{\mathbf{e}} \pi_{\mathbf{r}}\left(\pi_{\mathbf{r}+\mathbf{e}}-1\right)\left[\sum_{k=0}^{\infty} \frac{(-1)^{k}}{k !} \pi_{\mathbf{r}+\mathbf{e}}^{k} a_{\mathbf{r}+\mathbf{e}}^{k}\right] a_{\mathbf{r}}\right\}
$$

where the factor in square brackets has been introduced to prevent new particles from being added 
at occupied sites. (A slightly more subtle way of expressing the same constraint can be found in Ref. [27]). The lowest order terms of the Lagrangian deriving from the Liouvillian (15) reproduce those of equation (14). In either case, a RG analysis of the Lagrangian produces a runaway from the trivial, diffusion-like fixed point corresponding to the first line of equation (14) in any dimension. While one may hope to obtain information about cluster growth by methods similar to those of Langer [28], we conclude that perturbation theory is not able to solve fully the problem of the Eden model.

\section{The DLA model.}

We formulate the DLA model as follows. There is a steady (but small) flow of diffusing (D) particles and an aggregate of A particles. At epoch $t_{0}=0$ there is just one A particle at the origin. If a $\mathrm{D}$ particle finds itself in a site which is nearest neighbour to a site containing $\mathrm{A}$ particles, it may turn into an A particle itself and stop, with a certain rate $\mu$. We may also impose the constraint that no more than one A particle may occupy any site. Yet it is commonly believed that this constraint is irrelevant, and that the statistical properties of DLA aggregates are the same, in presence or in absence of this constraint, modulo a rescaling of the fundamental length. We shall therefore only consider the " penetrable " model without constraint in what follows.

The reaction scheme defining the model is therefore

$$
\begin{gathered}
\mathrm{D}_{\mathbf{r}} \stackrel{\lambda}{\longrightarrow} \mathrm{D}_{\mathbf{r}+\mathbf{e}}, \\
\mathrm{D}_{\mathbf{r}}+\mathrm{A}_{\mathbf{r}+\mathbf{e}} \stackrel{\mu}{\longrightarrow} \mathrm{A}_{\mathbf{r}}+\mathrm{A}_{\mathbf{r}+\mathbf{e}} .
\end{gathered}
$$

The corresponding Liouvillian reads :

$$
L=\sum_{\mathbf{r}}\left\{\lambda \sum_{\mathbf{e}}\left(\pi_{\mathbf{r}+\mathbf{e}, \mathbf{D}}-\pi_{\mathbf{r}, \mathbf{D}}\right) a_{\mathbf{r}, \mathbf{D}}+\mu \sum_{\mathbf{e}}\left(\pi_{\mathbf{r}, \mathbf{A}}-\pi_{\mathbf{r}, \mathbf{D}}\right) \pi_{\mathbf{r}+\mathbf{e}, \mathbf{A}} a_{\mathbf{r}+\mathbf{e}, \mathbf{A}} a_{\mathbf{r}, \mathbf{D}}\right\} .
$$

We have not written down explicitly the source at infinity producing the steady income of D particles. This Liouvillian corresponds to the Lagrangian

$$
\mathfrak{L}=\sum_{\mathbf{r}}\left\{\bar{\phi}_{\mathbf{r}} \dot{\phi}_{\mathbf{r}}+\bar{\psi}_{\mathbf{r}} \dot{\psi}_{\mathbf{r}}-\lambda \sum_{\mathbf{e}}\left(\bar{\psi}_{\mathbf{r}+\mathbf{e}}-\psi_{\mathbf{r}}\right) \psi_{\mathbf{r}}-\mu \sum_{\mathbf{e}}\left(\bar{\phi}_{\mathbf{r}}-\bar{\psi}_{\mathbf{r}}\right)\left(1+\phi_{\mathbf{r}+\mathbf{e}}\right) \phi_{\mathbf{r}+\mathbf{e}} \psi_{\mathbf{r}}\right\} .
$$

where $\bar{\psi}, \psi$ refer to D particles, and $\bar{\phi}, \phi$ to A ones. This Lagrangian was introduced in reference [24], where it was remarked that the mean field theory of DLA propounded by Witten and Sander [2] and by Nauenberg [29] could be recovered by neglecting the terms proportional to $\bar{\phi}_{\mathbf{r}+\mathbf{e}}$.

It is easy to reintroduce impenetrability along the lines of the previous section : as we have mentioned, this should not have important effects on the asymptotic behaviour of the model. On the other hand, since $\mathfrak{l}$ is linear in the $\bar{\psi}, \psi$ fields, they may be integrated out. One obtains a deterministic equation for $\psi$ :

$$
\dot{\psi}_{\mathbf{r}}=\lambda \sum_{\mathbf{e}}\left(\psi_{\mathbf{r}+\mathbf{e}}-\psi_{\mathbf{r}}\right)-\mu\left[\sum_{\mathbf{e}}\left(1+\varphi_{\mathbf{r}+\mathbf{e}}\right) \phi_{\mathbf{r}+\mathbf{e}}\right] \psi_{\mathbf{r}} .
$$

In the limit $\mu \rightarrow \infty$, if the incoming flow is sufficiently small, $\psi$ must vanish on the aggregate and satisfy Laplace's equation otherwise. One recovers therefore the Laplacian description of DLA suggested by reference [30]. By the same token, one is able to recover the nonlocal field theory of DLA contained in reference [19]. 
On the other hand, it is much more difficult to go beyond the first step. The field theory which one obtains never becomes critical, much for the same reasons as the Eden model. A naive RG approach leads to runaway from the trivial (diffusion-like) fixed point in any dimension. It is hard to make a link with the deterministic growth equations of Sander et al. [31] because of the presence in our equations of a diffusion " halo " which these authors have cut down with some arbitrariness. Nevertheless the derivation of a field theoretical model of DLA is in our opinion a necessary step for further investigations.

\section{Discussion.}

Whereas the kinematic part of the derivation of field theories of these aggregation processes can be regarded as solved, one has made little program in understanding their dynamics. This may be due to the lack of an exactly solvable field theoretical model which captures the essential physics and from where a perturbative approach can be started. It is also possible however that one has to reconsider the whole $R G$ approach to aggregation phenomena, especially in view of the analysis made by Halsey et al. [8] of the coexistence of several singularity populations in DLA clusters. Such a phenomenon corresponds to the existence of an infinite set of relevant parameters around a RG fixed point. This behaviour has been observed by Kim et al. [32] in the field theoretical description of conduction near the percolation threshold and in a controversial paper by Kravtsov and Lerner [33] on the scaling theory of two dimensional localization. Their phenomenological counterparts are the anomalous dispersion of voltage distribution in percolating clusters [34] and of the participation ratio at the localization threshold [35] respectively. It is interesting to remark that while the infinite set of relevant parameters has been spotted in field theories at the critical dimension, its phenomenological consequence appears to be ubiquitous. If such is the case of DLA one would need a deeper understanding of RG to get an explanation of the stability of this phenomenon.

Note added in proof : Field theory of aggregation processes have been independently introduced along lines parallel to those of the present work by D. Elderfield (J. Phys. A 18(1985) L-773).

\section{References}

[1] Eden, M., in Proceedings of the Berkeley Symposium on Mathematical Statistics Probability, J. Neyman ed. (Berkeley : University of California Press) 1961. Vol. IV, p. 223.

[2] Witten, T. A. and Sander, L. M., Phys. Rev. Lett. 47 (1981) 1400 ; Phys. Rev. B 27 (1983) 5686.

[3] Family, F. and Landau, D. P. (eds.), Kinetics of Aggregation and Gelation (Amsterdam : NorthHolland) 1984.

[4] Parisi, G. and Zhang, Y. C., Phys. Rev. Lett. 53 (1984) 1791.

[5] Vannimenus, J., Nickel, B. and Hakim, V., Phys. Rev. B 30 (1984) 391.

[6] Pietronero, L., communication at the CECAM Workshop on kinetics of Aggregation and Gelation, Sept. 1984 unpublished.

[7] Turkevitch, L. and Sher, Standard Oil Cỏmpany preprint (1985).

[8] Halsey, T. C., Jensen, M. H., Kadanoff, L. P., Procaccia, I. and Shraiman, B. I., University of Chicago preprint (1985).

[9] Амтт, D. J., Field Theory the Renormalization Group a Critical Phenomena, 2nd. ed. (Singapore : World Scientific) 1985.

[10] SCHLÖGL, F., Z. Phys. 253 (1972) 147.

[11] Amati, D., Ciafaloni, M., Marchesini, G. and Parisi, G., Nucl. Phys. 448 (1976) 483.

[12] Grassberger, P. and De La Torre, A., Ann. Phys. (N.Y.) 122 (1979) 373.

[13] Cardy, J. L. and Sugar, R. L., J. Phys. A 13 (1980) L-423. 
[14] JansSen, H. K., Z. Phys. B 42 (1981) 151.

[15] Cardy, J. L., J. Phys. A 16 (1983) L-709.

[16] Obukhov, S. P. and Peliti, L., J. Phys. A 16 (1983) L-167.

[17] Cardy, J. L. and Grassberger, P., J. Phys. A 18 (1985) L-267.

[18] Janssen, H. K., Z. Phys. B 58 (1985) 311.

[19] Parisi, G. and Zhang, Y. C., J. Stat. Phys. (1985).

[20] Shapir, Y. and Zhang, Y. C., J. Physique Lett. (1985).

[21] ShaPIR, Y., Brookhaven preprint (1985).

[22] DoI, M., J. Phys. A 9 (1976) 1465.

[23] Grassberger, P. and Scheunert, M., Fortschr. Phys. 28 (1980) 547.

[24] Peliti, L., J. Physique 46 (1985) 1469.

[25] Martin, P. C., Siggia, E. D. and Rose, H. A., Phys. Rev. A 8 (1973) 423.

[26] Schulman, L. S., Techniques and Appliçations of Path Integration (New York : J. Wiley) 1981.

[27] Peliti, L., Lecture at the Cargèse Summer School " On Growth and Forms », 1985, to be published.

[28] LONGER, J. S., Rev. Mod. Phys. 52 (1980) 1.

[29] Nauenberg, M., Phys. Rev. B 78 (1983) 449.

[30] Niemeyer, L., Pietronero, L. and Weissman, H., Phys. Rev. Lett. 52 (1984) 1033.

[31] SANDER, L. M., Ramanlal, P. and Ben-JACOB, E., University of Michigan preprint, 1985.

[32] Harris, A. B., Kim, S. and Lubensky, T. C., Phys. Rev. Lett. 53 (1984) 743.

[33] Kravtsov, V. E. and Lerner, I. V., Solid State Commun. 52 (1984) 593.

[34] De Arcangelis, L., Redner, S. and Coniglio, A., Phys. Rev. B 31 (1985) 4725.

[35] Ioffe, L. B., SAgdeev, I. R. and Vinokur, V. M., J. Phys. C 18 (1985) L-641. 